\title{
NOBEL PRIZES FOR 1955
}

$\mathrm{T}$ HE following awards of Nobel Prizes to scientific men have been announced:

\section{Physics: Profs. P. Kusch and W. E. Lamb}

The joint award of the Nobel Prize for Physics for 1955 to Prof. P. Kusch, of Columbia University, and Prof. W. E. Lamb, of Stanford University, recognizes a common feature in their contributions to physics. Both have applied the methods of high-precision radio-frequency spectroscopy to atomic phenomena, in which the effect on the electrons of their own radiation field, though very small, is significant.

Prof. Kusch has applied the methods of radiofrequency resonance in atomic and molecular beams to determine, with the highest achievable precision, the magnetic moment of atomic nuclei, and of the electron. In this way he has shown that the spinmoment of the electron is not precisely one Bohr magneton, but (1.001146 \pm 0.000012$)$ Bohr magnetons. Modern theories, developed at about the same time, and greatly stimulated by the experimental work of Kusch and of Lamb, predict tho value $1+e^{2} / h c-11 \cdot 89 e^{4} / h^{2} c^{2}+\ldots=1 \cdot 00114536$. Kusch's measurements of nuclear magnetic moments, from nuclear resonance, and from atomic hyperfine structure, show small but significant discrepancies which throw light on the structure of atomic nuclei.

Prof. Lamb, while primarily a theoretical physicist, has turned to experiment to test the prediction of modern theories of quantum electrodynamics, that the fine structure of the hydrogen spectrum is modified by the reaction of the electron with its radiation field. According to the simple Dirac wave equation, the energy of the metastable $2 s \frac{1}{2}$ level of hydrogen is exactly equal to the energy of the $2 p \frac{1}{2}$ level. In an experiment where transitions are induced between these two levels in an atomic beam, Lamb demonstrated that they are separated by $1057 \cdot 77 \pm$ $0.10 \mathrm{Mc} / \mathrm{s}$. Modern theories predict 1057.19 $0.16 \mathrm{Mc} / \mathrm{s}$.

Prof. Lamb has recently accepted appointment to the chair of theoretical physics in Oxford.

\section{Physiology and Medicine: Prof. Hugo Theorell}

The Nobel Prize for Physiology and Medicine for 1955 has been awarded to Prof. Hugo Theorell, director of the Biochemical Department of the Medical Nobel Institute of Stockholm, for his work on flavoprotein enzymes and hæmoproteins such as cytochrome $c$, peroxidase, catalase and myoglobin. The study of the "old flavoprotein enzyme", the prosthetic group of which is flavin monnnueleotide (FMN), was carried out by him when he was holding a Rockefeller Foundation Fellowship at the Kaiser Wilhelm Institute (now Max Planck Institute) for Cell Physiology (Berlin-Dahlem). It is the electrophoretic separation of this enzyme from a poly. saccharide, which accompanied it, that enabled him to purify this enzyme and to determine its main properties. Of special interest, however, is his extensive study of cytochrome $c$, the extractable and soluble component of the cytochrome system which constitutes the intracellular respiratory chain of catalysts linking the dehydrogenase systems with molecular oxygen, thus providing the essential path for hydrogen or electron transfer from the activated molecules of substrates to oxygen. To the study of the physico-chemical properties of cytochrome $c$ and other hæmoproteins, the mechanism of the reactions in which they are involved and the nature of the links between their prosthetic groups and protein, Prof. Theorell has applied great technical skill and a variety of modern physico-chemical and biochemical methods.

More recently, Prof. Theorell and his co-workers studied the properties of alcohol dehydrogenase extracted and purified from liver, and they were able for the first time to follow spectrophotometrically the kinetics of reactions between the enzyme and the reduced co-enzyme I (diphosphopyridine nucleotide). From this work emerged a method for the precise estimation of minute amounts of alcohol, which is of great value for medico-legal work. Prof. Theorell has established a very active centre of research which attracted many workers from different countries to his department which, in 1948, was transferred to the new building of the Medical Nobel Institute, where it is provided with all facilitios for the study of a great variety of biophysical and bio. chemical problems.

\section{Chemistry: Prof. Vincent du Vigneaud}

This year's Nobel Prize for Chemistry goes to Prof. Vincent du Vigneaud, professor and head of the Department of Biochemistry, Cornell University Medical College, New York. Prof. du Vigneaud had many achievements to his name before his recent outstanding work on the structure and synthesis of oxytocin and vasopressin. His researches on the chemistry of insulin were a valuable early contribution in this difficult subject, and were naturally related to his studies on intermediary metabolism and sulphur-containing substances. A distinguished series of investigations on the mechanism of transmethylation and the biosynthesis of choline were based on his substantial experience of both chemical and biochemical methods and greatly added to our knowledge of the metabolism of methionine and of cystine. During the Second World War, Dr. du Vigneaud turned his attention to the chemistry of penicillin, and his synthesis of this substance was a significant contribution to this subject. More recently his activities, and those of his colleagues, have been largely directed to the isolation, chemistry and synthesis of the posterior pituitary hormones, oxytocin and vasopressin. The demonstration that these substances are closoly related octapeptides, each containing a disulphide bond spanning six amino-acid residues (a type of structure also found in insulin by Sanger), and the fact that, as long ago as 1935, du Vigneaud had shown that oxytocin can be reduced and reoxidized without obvious inactivation, paved the way to the synthesis of both of these hormones. Each step in a series of complex investigations has been checked with the utmost care, and the complete synthesis of these two related substances has opened wide the door to vast new developments in the pharmacology of peptide hormones and their chemical relations. 